E-Note

\title{
A Porous Convection Model for Grass Patterns
}

\author{
Sally E. Thompson ${ }^{1, *}$ and Karen E. Daniels ${ }^{2}$
}

1. Nicholas School of the Environment, Duke University, Durham, North Carolina 27707; 2. Department of Physics, North Carolina State University, Raleigh, North Carolina 27695

Submitted May 18, 2009; Accepted September 28, 2009; Electronically published November 12, 2009

ABSTRACT: Spatial ecological patterns are usually ascribed to Turingtype reaction-diffusion or scale-dependent feedback processes, but morphologically indistinguishable patterns can be produced by instabilities in fluid flow. We present a new hypothesis that suggests that fluid convection and chill damage to plants could form vegetation patterns with wavelengths $\approx 1-2$ times the plant height. Previous hypotheses for small-scale vegetation pattern formation relied on a Turing process driven by competition for water, which is thought to occur in large vegetation patterns. Predictions of the new hypothesis were consistent with properties of natural grass patterns in North Carolina, contradicting the Turing hypothesis. These results indicate that similarities in pattern morphology should not be interpreted as implying similarities in the pattern-forming processes, that small-wavelength vegetation patterns may arise from mechanisms that are distinct from those generating long-wavelength vegetation patterns, and that fluid instabilities should be recognized as a cause of ecological patterns.

Keywords: ecological patterns, thermal convection, soil moisture, chill damage.

\section{Introduction}

Ecological pattern formation occurs in a wide array of contexts (Rietkerk and van de Koppel 2008), but the hypothesized mechanisms have focused on scale-dependent feedback or reaction-diffusion processes. Mechanisms of this sort, where growth of the pattern is enhanced at small scales but suppressed at large scales, produce what are known as Turing patterns (Turing 1952). An alternative class of pattern-forming mechanisms known as hydrodynamic processes arise from instabilities in the motion of fluids, including both air and water (Cross and Hohenberg 1993). These processes are largely omitted from discussions of ecological patterns. Patterns arising because of Turing processes or fluid instabilities are not generally distinguishable on the basis of morphology alone.

Short-wavelength $(\lambda)$ vegetation patterns comprising brown and green bands with $\lambda \approx 10 \mathrm{~cm}$ have been ob-

\footnotetext{
* Corresponding author; e-mail: set8@duke.edu.
}

Am. Nat. 2010. Vol. 175, pp. E10-E15. (C) 2009 by The University of Chicago. 0003-0147/2010/17501-51280\$15.00. All rights reserved.

DOI: $10.1086 / 648603$ served in short-canopy warm-season grasses (von Hardenberg et al. 2001; Meron et al. 2004; G. L. Miller, personal communication); these were previously hypothesized to arise because of Turing-type effects based on water scarcity (as per Valentin et al. 1999). Thus, they would form part of a scale-invariant spectrum of vegetation patterning along with patterns of $\lambda \approx 50-100 \mathrm{~m}$ that commonly occur in deserts (Rietkerk et al. 2004; Deblauwe et al. 2008). The small-scale grass patterns are morphologically similar to both Turing patterns and those produced by thermal convection of a fluid (Cross and Hohenberg 1993; Shattuck et al. 1995).

We present a new hypothesis to explain these patterns: A thermal gradient between warm ground and cool air is unstable to thermal convection, generating a pattern of rising warm air and falling cold air within the grass canopy. Chilling injuries associated with the falling cold air then produce characteristic brown bands of "dead" vegetation. This hypothesis is consistent with several features of smallscale vegetation patterns, including their length scale, rapid onset, and transient nature. For a Turing process driven by soil moisture availability to generate coherent, smallscale patterns it would require a high degree of organization within the soil. Such organization would be remarkable, given the tendency of soil moisture redistribution and root activity to homogenize soil moisture over short length scales (Katul et al. 1997). In this article, we draw on established models of thermal convection to predict the conditions under which convection rolls might lead to patterning in a vegetation canopy. These quantitative predictions are then tested against field observations of a naturally occurring instance of grass patterning.

\section{Thermal-Convection Hypothesis}

When a fluid-saturated porous medium is heated from below, heat is transported by thermal conduction. Although this heating results in the base layer of fluid being more buoyant than the overlying fluid, both viscous drag and thermal diffusivity oppose its upward motion. As the temperature at the base of the fluid column increases, the 
buoyancy overcomes these stabilizing effects and the conducting regime gives way to convection. The convective state is characterized by regions of rising, warm fluid adjacent to regions of falling, cold fluid (e.g., Ball 2001). The resulting convection rolls are shown schematically in figure 1, along with a definition of key parameters. Typically, convection rolls form locally parallel stripes (which can bend to form labyrinthine patterns) or spots; in the case of convection within a porous medium (Howle et al. 1993; Shattuck et al. 1995), the patterns are morphologically similar to those observed in vegetation.

We hypothesize that small-scale grass patterns can be formed by this convective process: the observed pattern of green and brown grasses could arise from the combination of convection and chill damage if falling air is cold enough to damage grass and rising air is warm enough to protect it. The rapid formation and transient nature of grass patterns is suggestive of chill damage as a possible cause: such damage occurs when ambient temperatures drop too rapidly for plants to acclimate, which usually results in nonfatal tissue damage such as browning and leaf death (Taiz and Zeiger 2005). Warm-season grasses can be damaged by less than an hour of exposure to nearfreezing temperatures (Atwell et al. 1999).

The onset of porous medium convection occurs when buoyancy overcomes the stabilizing forces of viscosity and heat conduction. This balance is quantified by a ratio known as the Rayleigh number (Ra), where the buoyancy terms are in the numerator and the stabilizing terms are in the denominator:

$$
\mathrm{Ra}=\frac{K \alpha g \Delta T d}{\kappa \nu} .
$$

Here, $\alpha$ is the thermal expansion coefficient of the fluid, $g$ is the acceleration due to gravity, $\Delta T$ is the difference in temperature between the solid bottom and the fluid at the upper boundary, $d$ is the thickness of the porous medium, and $\nu$ is the kinematic viscosity of the fluid. Two parameters, the permeability $K$ and the thermal conduc- tivity $\kappa$, depend on the details of the porous medium, including the porosity $\phi$ and the geometry of the pores. When $\mathrm{Ra}$ exceeds a critical threshold $\mathrm{Ra}_{\mathrm{c}}$, convection begins (Lapwood 1948). In an isotropic medium, the critical Rayleigh number is given by $\mathrm{Ra}_{\mathrm{c}}=4 \pi^{2}$. However, the permeability of the voids in a vegetation canopy depends on whether the fluid is moving vertically (parallel to the grass blades) or horizontally (intercepting multiple grass blades). Thus, the canopy is anisotropic and the Rayleigh number must be redefined to account for directional effects:

$$
\mathrm{Ra}_{\mathrm{c}}=\pi^{2}\left[\left(\frac{\kappa_{\perp} / \kappa_{\|}}{K_{\perp} / K_{\|}}\right)^{1 / 2}+1\right]^{2} .
$$

The subscripted parameters (see fig. 1) indicate the material properties that are parallel and perpendicular to the temperature gradient; $K_{\|}$and $\kappa_{\|}$are used in equation (1) in place of the average values (Straus 1974; Straus and Schubert 1978; Kvernvold and Tyvand 1979).

A grass canopy may be modeled as a series of upright cylindrical rods (grass blades) of radius $a$ that are spaced a distance $2 b$ apart, as shown in figure 1. Using this approximation, we can estimate the permeabilities $K_{\|}$and $K_{\perp}$ using the approach of Happel (1959). The permeability in the lateral direction $K_{\perp}$ can be computed directly from the geometry:

$$
K_{\perp}=\frac{b^{2}}{4}\left(\ln \frac{b}{a}-\frac{1}{2} \frac{b^{4}-a^{4}}{b^{4}+a^{4}}\right) .
$$

To estimate the vertical permeability $K_{\|}$, the CarmenKozeny equation for $K$ is equated to the solution for viscous flow about a cylinder, to give $K_{\|}=\phi \mathrm{m}^{2} / k_{\|}$. The geometric parameter $m$ is analogous to the hydraulic radius and is given by $m=\left(b^{2}-a^{2}\right) / 2 a$. The Kozeny constant $k_{\|}$parameterizes the morphology of the porous medium and is estimated (Happel 1959) as

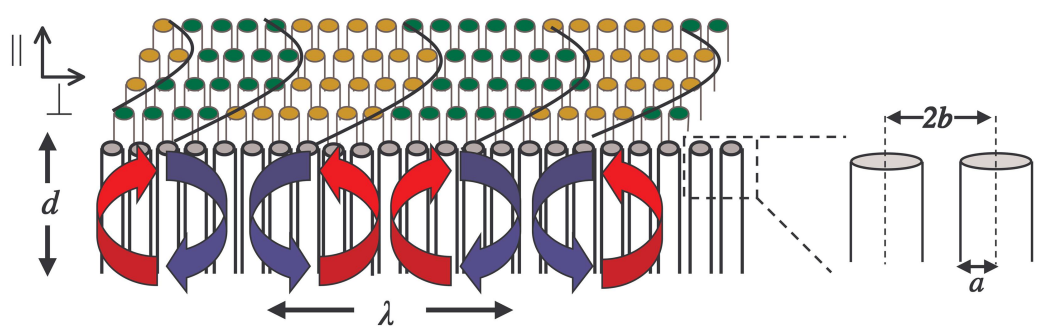

Figure 1: Schematic of parameters used in porous convection model (not to scale). 


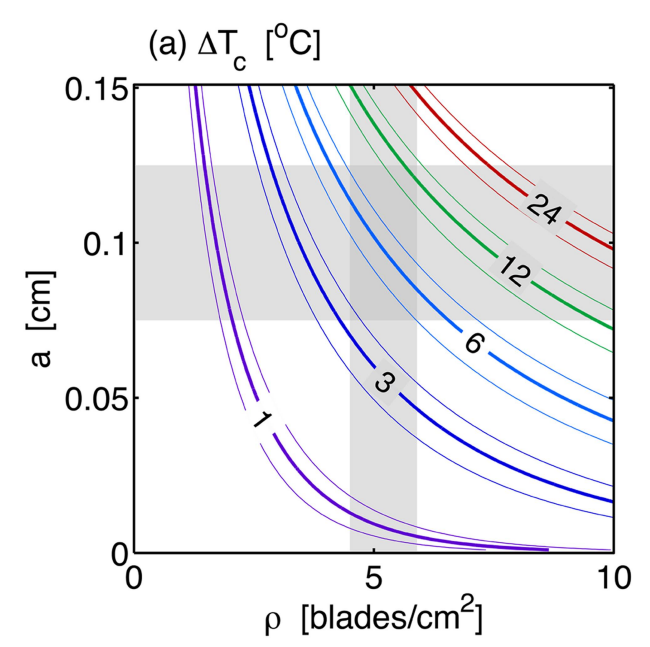

(b) $\lambda_{c} / d$

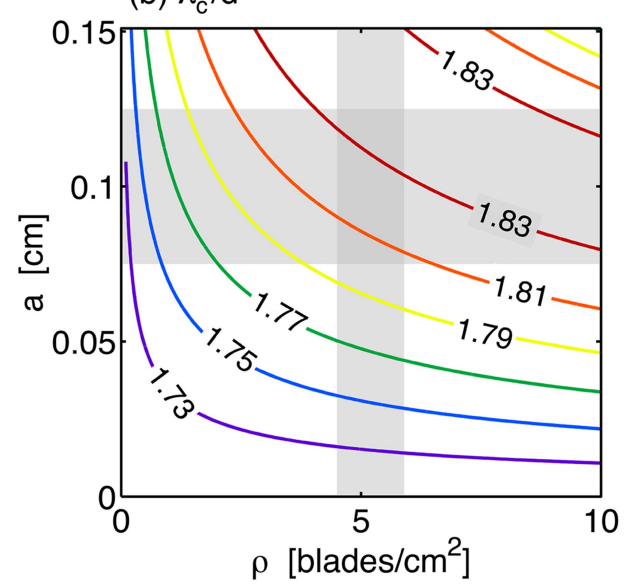

(c) $\Delta \mathrm{T}_{\mathrm{c}}\left[{ }^{\mathrm{O}} \mathrm{C}\right]$

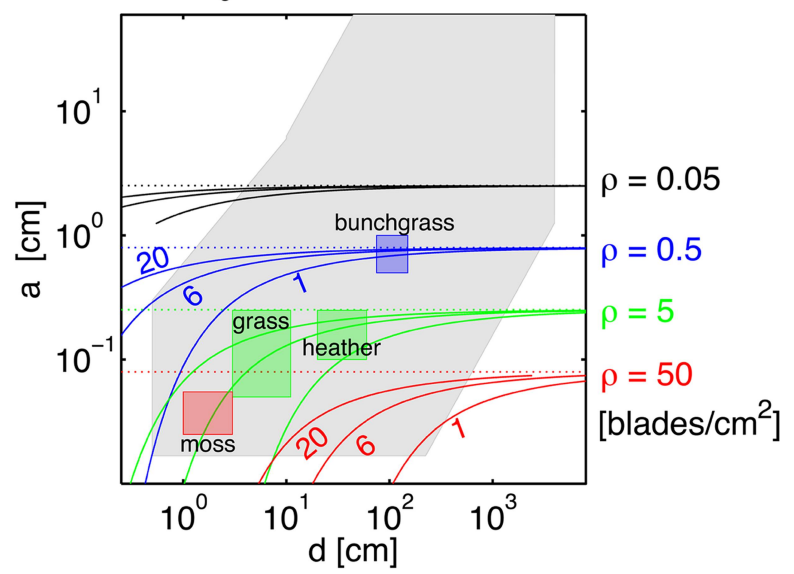

Figure 2: Contour plots of numerically computed values of the critical temperature difference $\Delta T_{c}$ and critical wavelength $\lambda_{c}$ for a range of appropriate vegetation sizes $(a, d)$ and densities $(\rho) . a, b$, Values computed for an observed lawn grass parameter regime (see fig. 3). Gray bars indicate uncertainty in stem radius $a$ and density $\rho$ measurements, while the thin curves associated with each contour of $\Delta T_{\mathrm{c}}$ indicate the uncertainty in the measurement of height $d$. $c$, Critical temperature difference

$$
k_{\|}=\frac{2 \phi^{3}}{(1-\phi)\left\{2 \ln [1 /(1-\phi)]-3+4(1-\phi)-(1-\phi)^{2}\right\}} .
$$

The porosity $\phi$ is determined from the grass blade radius $a$ and blade density $\rho$ via the relation $\phi=1-\rho\left(\pi a^{2}\right)$. To estimate the thermal diffusivity of the grass canopy, we average the component materials (water, grass) in proportion to their volumetric presence $(\phi$ and $(1-\phi)$, respectively), assuming that grass is composed of $85 \%$ water and $15 \%$ cellulose. Given the high porosity of the canopy, we assume that $\kappa_{\|} \approx \kappa_{\perp}$. Thus, for known or measurable properties of a grass canopy, it is possible to calculate the value of $\Delta T_{\mathrm{c}}$ required for the onset of convection ( $\mathrm{Ra}>$ $\left.R a_{c}\right)$.

For anisotropic media, the critical wave number of the convection pattern at onset (Kvernvold and Tyvand 1979) is

$$
q_{\mathrm{c}}=\frac{\pi}{d}\left(\frac{K_{\perp}}{K_{\|}} \frac{\kappa_{\perp}}{\kappa_{\|}}\right)^{-1 / 4} .
$$

On the basis of this analysis, we can determine the necessary conditions for the convection-chill hypothesis to hold given measured values of $a, d, \rho$, and ambient meteorological conditions. The requirements are a large enough temperature gradient $\left(\Delta T>\Delta T_{\mathrm{c}}\right)$, an air temperature that is cold enough (e.g., $\lessgtr 0^{\circ} \mathrm{C}$ ) to cause chill damage, and no wind or turbulence to disrupt convection. Second, we can predict the expected pattern wavelength given these conditions and compare this prediction with observations. Figure $2 a, 2 b$ shows the dependence of $\Delta T_{c}$ and wavelength $\lambda_{\mathrm{c}}=2 \pi d / q_{\mathrm{c}}$ on $a$ and $\rho$. While $\Delta T_{\mathrm{c}}$ is quite sensitive to the particular porosity (set by $a$ and $\rho$ ), $\lambda_{c} \approx 1.8 d$ over the full range of reasonable characteristics.

\section{Field Observations}

Grass patterning with a labyrinthine morphology and wavelength $\lambda \approx 10 \pm 2 \mathrm{~cm}$ was fortuitously observed on a Bermuda grass lawn (Cynodon dactylon $\times$ Cynodon transvaalensis Burtt-Davy var. Patriot) at Duke University

computed as a function of $a$ and $d$ for a range of realistic vegetation densities: $\rho_{\text {moss }} \approx 50$ blades $\mathrm{cm}^{-2}($ red $), \rho_{\text {grass, heather }} \approx 5$ blades $\mathrm{cm}^{-2}$ (green $)$, $\rho_{\text {bunchgrass }} \approx 0.5$ blades $\mathrm{cm}^{-2}$ (blue), very low $\rho=0.05$ blades $\mathrm{cm}^{-2}$ (black). For each $\rho$, contours show $\Delta T_{\mathrm{c}}=1^{\circ}, 6^{\circ}$, and $20^{\circ} \mathrm{C}$, and shaded rectangles denote the approximate range of $a$ and $d$ values for the corresponding vegetation type (indicated by color). Gray shaded area indicates a realistic regime for terrestrial plants based on typical scaling laws for woody and nonwoody species (Rich et al. 1986). 
(Durham, NC) on November 12, 2008. The relevant geometric factors required to calculate the critical Rayleigh number $\mathrm{Ra}_{\mathrm{c}}$ (eq. [1]) were measured in situ and confirmed using high-resolution photographs of the patterns, which allowed us to estimate $\Delta T_{\mathrm{c}}$ and $\lambda_{\mathrm{c}}$ for the lawn. Meteorological data were obtained for the night of November 10 , the only recent preceding night when a frost occurred. The 2-day delay corresponds to the typical time frame for symptoms of chill damage to appear (Atwell et al. 1999). Meteorological data were obtained from the North Carolina State Climate Service (North Carolina State Climate Office 2008) for the six weather stations surrounding Durham that report both ground and air temperatures (stations SILR, REED, OXFO, KTDF, KIGX, and KRDU) and averaged to obtain an estimate of $\Delta T$. Wind data were obtained from sonic anemometer instrumentation overlying a 50-cm-tall grass field located at the Duke Forest, approximately $16 \mathrm{~km}$ from the patterned site (see Novick et al. 2005 for site details). Immediately after observing the patterns, we measured volumetric soil-moisture $(\theta)$ content in the root zone (top $12 \mathrm{~cm}$ ), taking a total of 52 measurements until rain commenced and further measurements were deemed unreliable.

\section{Results}

The measured geometric properties of the lawn were: grass-blade radius $a=0.1 \pm 0.025 \mathrm{~cm}$, canopy depth $d=6 \pm 1 \mathrm{~cm}$, and lawn density $\rho=5.2 \pm 0.7$ blades $\mathrm{cm}^{-2}$. These values are typical for dense lawns (Brede 1999; Hamilton and Waddington 1999). As shown in figure $2 a$, $2 b$, the use of these values in the thermal convection model predicted values of $\Delta T_{\mathrm{c}}=6.3^{\circ} \pm 3.6^{\circ} \mathrm{C}$ and $\lambda_{\mathrm{c}}=$ $10.9 \pm 1.8 \mathrm{~cm}$. The wavelength of the pattern was measured from photographs to be $\lambda=10 \pm 2 \mathrm{~cm}$, in agreement with the model results for the measured lawn parameters. Figure 3 shows all measured field parameters.

The meteorological data showed that on November 10 , 2008 , ground temperatures dropped from $12.6^{\circ} \mathrm{C}$ to $11.3^{\circ} \mathrm{C}$ from midnight to 7 a.m., and air temperatures dropped from $4.3^{\circ} \mathrm{C}$ to $-0.4^{\circ} \mathrm{C}$ over the same period. Thus, $\Delta T$ ranged from $8.2^{\circ} \mathrm{C}$ to $12^{\circ} \mathrm{C}$ throughout the night, with the greatest $\Delta T$ coinciding with the coldest air temperatures close to dawn. An uncertainty of $2{ }^{\circ} \mathrm{C}$ is associated with the measured $\Delta T$ due to variation across the six weather stations; however, even when this is incorporated, the ambient $\Delta T$ exceeded the model estimate for $\Delta T_{c}$. Wind conditions overnight were calm. The vertical momentum flux was $3 \times 10^{-3} \mathrm{~m}^{2} \mathrm{~s}^{-2}$, and the horizontal shear velocity was $0.06 \mathrm{~m} \mathrm{~s}^{-1}$, indicating that the potential for production of turbulence near ground level was negligible.

Two examples of the soil water content $(\theta)$ measurements as a function of brown/green locations within the pattern are shown in figure 3. No correlation between grass condition and $\theta$ could be discerned. All moisture readings exceeded the permanent wilting point $\theta^{*}$ at which plants begin to experience water stress. The mean $\theta$ for the brown patches was $\bar{\theta}_{\text {brown }}=41.4 \%$, which was slightly lower than $\bar{\theta}_{\text {green }}=44.3 \%$. This difference is comparable to the error in the instrument, and it was not statistically significant $(P=.24)$, nor, given that $\bar{\theta}>\theta^{*}$, was it large enough to be physiologically important (Rodriguez-Iturbe and Porporato 2004).

\section{Discussion and Conclusions}

On the basis of the observations of this occurrence of grass patterns, all data (the actual air temperature $\Delta T$ exceeding the critical air temperature $\Delta T_{c}$, the wavelength $\lambda$ approximating the critical wavelength $\lambda_{c}$, still-air conditions, no significant differences in water content $\bar{\theta}$ between green and brown bands, and all water contents exceeding the wilting point $\theta^{*}$ ) support the interpretation that convection and chill damage generated patterning. An unusually large $\Delta T$ value two nights before the observation exceeded the predicted value of $\Delta T_{\mathrm{c}}=6.3^{\circ} \pm 3.6^{\circ} \mathrm{C}$ and coincided with air temperatures near freezing, which are low enough to cause chill damage to plants, but ground temperatures that were sufficiently warm to generate convective motion within the grass canopy and locally buffer grass from chill damage. Still atmospheric conditions suggest that thermal convection, and not wind, was the major driving force for flow within the grass canopy.

The porous convection model is consistent with the grass patterning observations, and it provides an explanation for the transience of observed grass patterns since chill damage is not fatal and plants recover rapidly. The infrequent observation of the phenomenon is explained by the specificity of the required nonbiological and biological factors coinciding with the presence of plants susceptible to chill damage. The length scales of the observed patterning, along with length scales reported by other authors for similar grass patterns (von Hardenberg et al. 2001), correspond well to predictions from the convection model. Thus, we find that the porous convection model explains multiple, independent aspects of the grass patterning phenomenon. Future laboratory experiments could be readily established that have the aim of comparing the predictions for the critical temperature difference and the pattern wavelength with experimental results. Such experiments could be performed in a constant-temperature cold room, using heating elements beneath a grass canopy to provide the temperature gradient; as such, $\Delta T_{c}$ could be measured as a function of blade height and density. Conversely, the lack of any discernible correlation between the grass pattern and the water content is contrary 


\section{E14 The American Naturalist}

(a)

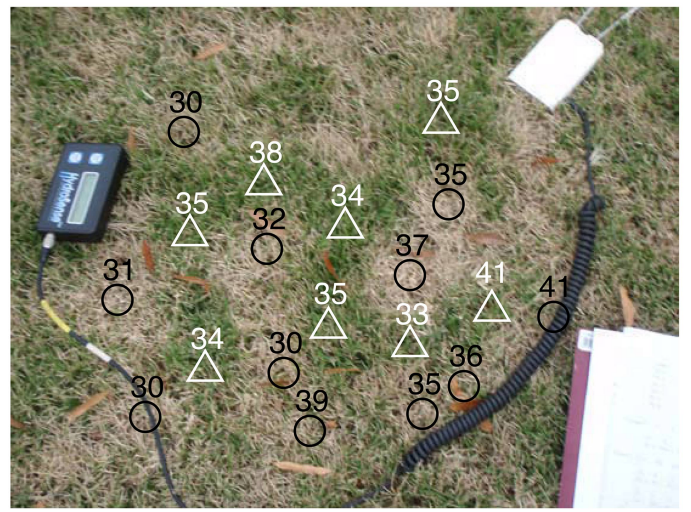

(b)

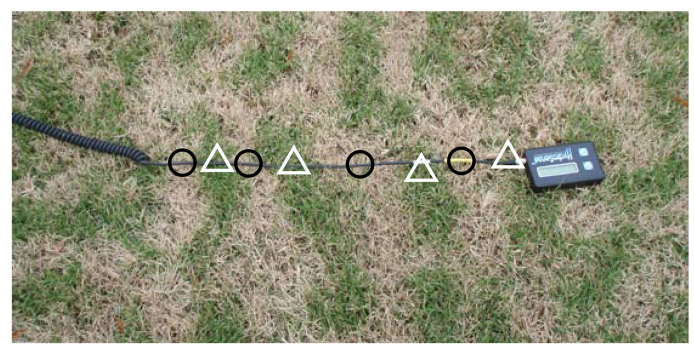

(c)

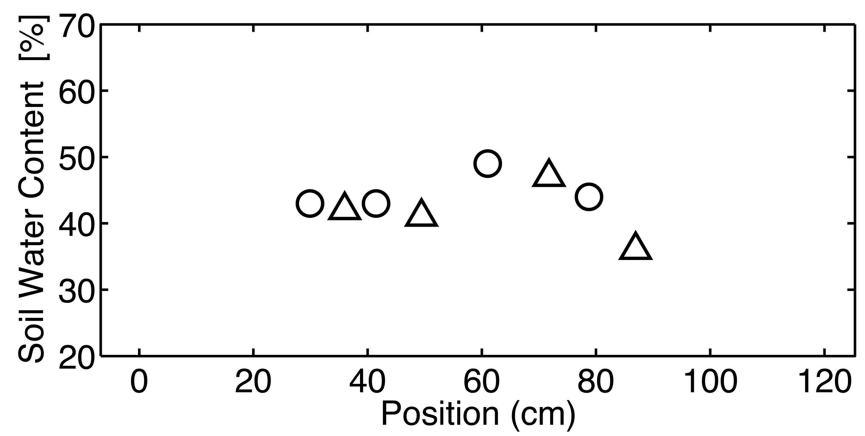

(d)

\begin{tabular}{l}
\multicolumn{3}{c}{$\begin{array}{c}\text { Measured Pattern and } \\
\text { Lawn Parameters }\end{array}$} \\
\begin{tabular}{|c|cc|}
\hline$\lambda$ & $10 \pm 2$ & $\mathrm{~cm}$ \\
\hline $\mathrm{d}$ & $6 \pm 1$ & $\mathrm{~cm}$ \\
\hline $\mathrm{a}$ & $0.1 \pm 0.025$ & $\mathrm{~cm}$ \\
\hline$\rho$ & $5.2 \pm 0.7$ & blades $/ \mathrm{cm}^{2}$ \\
\hline
\end{tabular}
\end{tabular}

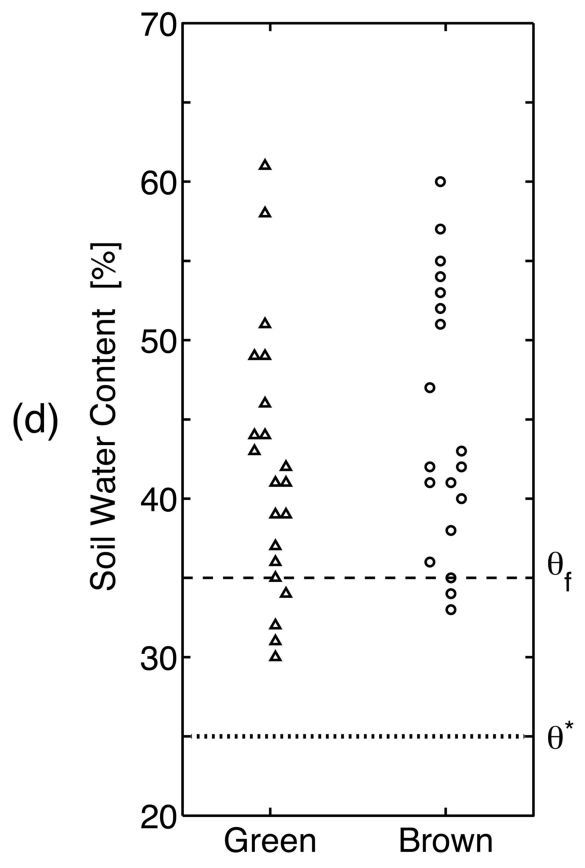

Figure 3: Sampling of soil water content $\theta$ at 52 points. $a$, Measurements of $\theta$ within a labyrinthine pattern, printed within image. $b$, Measurements of $\theta$ along a transect perpendicular to a striped pattern, plotted in $c$. $d$, All $52 \theta$ measurements made on the site and sorted by brown/green location. Dashed line $\theta_{\mathrm{f}}$ indicates the field capacity, and dotted line $\theta^{*}$ indicates the wilting point (Rodriguez-Iturbe and Porporato 2004). Parameters used to calculate critical temperature difference $\Delta T_{\mathrm{c}}$ and critical wavelength $\lambda_{\mathrm{c}}$ for grass canopy are listed in upper right corner.

to the predictions of a Turing model based on water availability. Unlike the Turing models (von Hardenberg et al. 2001; Rietkerk et al. 2002), the bifurcation to a patterned state is a simple, forward bifurcation with no bistability. Because chill damage does not change the porous properties of the vegetation, there is no feedback between the pattern and the fluid flow, and, thus, no hysteresis.

The thermal convection mechanism could apply to a range of vegetation types, as shown in figure $2 c$, but in practice a realistic regime of terrestrial plants (gray shading) coincides with reasonable temperature differences $\left(\Delta T_{c} \approx 6^{\circ} \mathrm{C}\right)$ in only a limited regime with stem density $1 \lesssim \rho \lesssim 10$ blades $\mathrm{cm}^{-2}$ and a small stem diameter
( $a \lesssim 1 \mathrm{~cm}$ ). For higher densities (e.g., mosses), $\Delta T_{\mathrm{c}}$ becomes unrealistically large. Where densities are low and canopies are tall, not only do wind-driven flows become more likely, but $\Delta T_{\mathrm{c}}$ can be very small and thus unlikely to cause any chill damage. A second consideration is that the vegetation must be of a type that is sensitive to chill damage. Warm-season grasses are ideal target species, but other ground-covering herbaceous species or even small, low-growing woody species could sustain this mechanism. Because our model applies to canopy geometries, which can be approximated by an array of cylinders, figure $2 c$ highlights mosses, lawn grasses, and bunch grasses. However, similar results would apply for other low and dense 
canopy geometries, such as heaths. More generally, we may conceive of other scenarios where pattern formation arising from fluid instabilities structures ecological communities. Convection can occur whenever buoyant fluid underlies denser fluid, such as in shallow lakes, where bottom sediments heat faster than overlying water; on lake or ocean floors subject to geothermal heating; or in strong salinity clines. Because convection results in efficient and spatially partitioned transport, it could establish microclimates that are relatively enriched or depleted in resources, with implications for the structure of organismal assemblages.

Convective mechanisms and other fluid instabilities resulting in pattern formation are morphologically indistinguishable from Turing processes, yet they result from fundamentally different mechanisms and imply different dynamics. The thermal convection/chill damage hypothesis appears to explain multiple aspects of observed shortwavelength vegetation patterns, and it suggests that ecological patterns can arise from hydrodynamic processes. Thus, three key issues emerge from these findings: the fallacy of equating pattern morphology to the mechanisms of pattern formation must be highlighted and avoided, small-wavelength vegetation patterns must be reconsidered as arising from mechanisms that are distinct from those generating long-wavelength vegetation patterns, and hydrodynamic mechanisms, including convection and other fluid instabilities, must be recognized as a potential cause of ecological patterns.

\section{Acknowledgments}

We thank the Clark group, N. George, H. Greenside, G. Katul, and S. Manzoni at Duke University for illuminating discussions and the loan of equipment. S.E.T. acknowledges support from the General Sir John Monash Foundation and the National Science Foundation (grants EAR0628342 and -063578).

\section{Literature Cited}

Atwell, B., P. Kriedemann, and C. Turner. 1999. Plants in action. Vol. 1. McMillan Education Australia, South Yarra, Victoria.

Ball, P. 2001. The self-made tapestry: pattern formation in nature. Oxford University Press, Oxford.

Brede, A. 1999. Turfgrass cultivars of Zoysia sinica. US Patent 5977450. Filed April 17, 1997; issued November 2, 1999.

Cross, M. C., and P. C. Hohenberg. 1993. Pattern formation out of equilibrium. Reviews of Modern Physics 65:851-1112.

Deblauwe, V., N. Barbier, P. Couteron, O. Lejeune, and J. Bogaert. 2008. The global biogeography of semi-arid periodic vegetation patterns. Global Ecology and Biogeography 17:715-723.

Hamilton, G., and D. Waddington. 1999. Infiltration rates on residential lawns in central Pennsylvania. Journal of Soil and Water Conservation 54:564-568.
Happel, J. 1959. Viscous flow relative to arrays of cylinders. American Institute of Chemical Engineering Journal 5:174-179.

Howle, L., R. P. Behringer, and J. Georgiadis. 1993. Visualization of convective fluid-flow in a porous medium. Nature 362:230-232.

Katul, G., P. Todd, and D. Pataki. 1997. Soil water depletion by oak trees and the influence of root water uptake on the moisture content spatial statistics. Water Resources Research 33:611-623.

Kvernvold, O., and P. A. Tyvand. 1979. Non-linear thermal-convection in anisotropic porous media. Journal of Fluid Mechanics 90: 609-624.

Lapwood, E. 1948. Convection of a fluid in a porous medium. Proceedings of the Royal Cambridge Philosophical Society 44:508521.

Meron, E., E. Gilad, J. von Hardenberg, M. Shachak, and Y. Zarmi. 2004. Vegetation patterns along a rainfall gradient. Chaos, Solitons and Fractals 19:367-376.

North Carolina State Climate Office. 2008. NC Climate Retrieval and Observations Network of the Southeast database. http://www.ncclimate.ncsu.edu/cronos. Accessed February 23, 2009.

Novick, K., P. Stoy, G. G. Katul, D. S. Ellsworth, M. Siqueira, J. Juang, and R. Oren. 2005. Carbon dioxide and water vapour exchange in a warm temperate grassland. Oecologia (Berlin) 138:259-274.

Rich, P. M., K. Helenurm, D. Kearns, S. R. Morse, M. W. Palmer, and L. Short. 1986. Height and stem diameter relationships for dicotyledonous trees and arborescent palms of Costa Rican tropical wet forest. Bulletin of the Torrey Botanical Club 113:241-246.

Rietkerk, M., and J. van de Koppel. 2008. Regular pattern formation in real ecosystems. Trends in Ecology \& Evolution 23:169-175.

Rietkerk, M., M. C. Boerlijst, F. van Langevelde, R. HilleRisLambers, J. van de Koppel, L. Kumar, H. H. T. Prins, and A. M. de Roos. 2002. Self-organization of vegetation in arid ecosystems. American Naturalist 160:524-530.

Rietkerk, M., S. C. Dekker, P. C. de Ruiter, and J. van de Koppel. 2004. Self-organized patchiness and catastrophic shifts in ecosystems. Science 305:1926-1929.

Rodriguez-Iturbe, I., and A. Porporato. 2004. Ecohydrology of watercontrolled ecosystems. Cambridge University Press, Cambridge.

Shattuck, M. D., R. P. Behringer, G. A. Johnson, and J. G. Georgiadis. 1995. Onset and stability of convection in porous-media: visualization by magnetic-resonance-imaging. Physical Review Letters 75:1934-1937.

Straus, J. M. 1974. Large-amplitude convection in porous media. Journal of Fluid Mechanics 64:51-63.

Straus, J. M., and G. Schubert. 1978. Existence of 3-dimensional convection in a rectangular box containing fluid-saturated porous media. Journal of Fluid Mechanics 87:385-394.

Taiz, L., and E. Zeiger. 2005. Plant physiology. Sinauer, New York.

Turing, A. M. 1952. The chemical basis of morphogenesis. Philosophical Transactions of the Royal Society B: Biological Sciences 237:37-72.

Valentin, C., J. M. d'Herbes, and J. Poesen. 1999. Soil and water components of banded vegetation patterns. Catena 37:1-24.

von Hardenberg, J., E. Meron, M. Shachak, and Y. Zarmi. 2001. Diversity of vegetation patterns and desertification. Physical Review Letters 87:198101-198104.

Associate Editor: Claire de Mazancourt Editor: Mark A. McPeek 